\title{
Valor predictivo de la pelvimetría clínica para el diagnóstico de la desproporción cefalopélvica de causa materna
}

\author{
Jaime Enrique Ruiz Sternberg, M.D.**; Germán Barón Castañeda, M.D.*
}

\begin{abstract}
RESUMEN: En las últimas décadas se ha visto cómo ha aumentado en forma progresiva el número de cesáreas por desproporción cefalopélvica de causa materna. En este trabajo se pretende rescatar la pelvimetría como medio clínico, auxiliar en el diagnóstico de esta entidad. Se realizaron mediciones de la pelvis en 145 pacientes, de las cuales 115 fueron incluidas en el presente estudio; de ellas 19 fueron llevadas a cesárea por desproporción. A todas las pacientes se les realizó medición de los diámetros biespinoso, bicrestíleo, bitrocantérico, Baudelocque, arcada subpúbica, bituberoso, palpación del promontorio sacro, de las espinas ciáticas, inclinación del sacro y paredes laterales de la pelvis. El promedio obtenido de los diferentes diámetros en la población de estudio fue menor que los valores aceptados en la literatura como normales. De todos los parámetros estudiados, sólo el diámetro bituberoso disminuido (p= 0.04$)$ y las paredes laterales convergentes $(p=0.007)$ tuvieron un valor predictivo. Se concluye que la pelvimetría es una herramienta útil que puede alertar al clínico, pero que a pesar de hallar alteraciones en la pelvimetría, todas las pacientes deben ser sometidas a una prueba de trabajo de parto y que sólo con alteraciones en su dinámica, se puede diagnosticar la desproporción cefalopélvica.
\end{abstract}

PALABRAS CLA VES: Desproporción cefalopélvica, biespinoso, bicrestíleo, bitrocantérico, Baudelocque, arcada, promontorio, sacro, espinas, bituberoso, paredes laterales.

SUMMARY: In the last decades there has been a progressive increase in the number of cesarean sections done for maternal cause cephalopelvic disproportion. This study is an atempt to rescue pelvimetry as a clinical media, helpful for the diagnosis of this entity. Measurements of the pelvis were done on 145 women, 115 of them included in this study; in 19, cesarean sections were practiced because of disproportion. In all of the patients the measurements taken were biespinous, bicrestilian, bitrocanteric and Baudelocque diameters, subpubic arch, promontory palpation, sciatic spines, sacrum concavity and lateral walls of the pelvis. The mean value for the diameters in the study group is less than the values accepted as normal in he literature. Of all the parameters studied, only the bituberous diameter ( $p=$ $0.04)$ and converging lateral walls $(p=0.007)$ had a predictive value. The conclusion is that pelvimetry is useful for alerting the obstetrician, but that even if alterations are found all patients should benefit of a labor trial, and that only alterations in the progression of labor can diagnose cephalopelvic disproportion.

KEY WORDS: Cephalopelvic disproportion; pelvimetry; biespinous, bicrestilian, bitrocanteric and baudelocque diameters; subpubic arch; promontory; sciatic spines; sacrura concavity; pelvic lateral walls.

En las últimas décadas se ha visto cómo ha aumentado en forma progresiva el índice de cesáreas con base en el diagnóstico de alteraciones en el trabajo de parto por desproporción cefalo-pélvica de causa materna. Así mismo, ha disminuido en forma muy importante el interés por el conocimiento de la arquitectura pelviana y de sus relaciones con la cabeza durante el trabajo de parto.

En la actualidad no existe un método preciso para el diagnóstico de este tipo de alteraciones antes del inicio del trabajo de parto y lo que se observa en la práctica clínica es que los obstetras no siguen un esquema ordenado de evaluación de la pelvis materna y suelen conferir mayor o menor valor a alguna de las medidas de forma arbitraria. Es por este motivo que con este trabajo se pretende hacer un análisis objetivo de la pelvimetría clínica como una herramienta útil en el diagnóstico anteparto de la desproporción cefalo-pélvica de causa materna.

\footnotetext{
* Coordinador Unidad de Investigación Clínica en reproducción humana del Hospintal Infantil L.V.S.

Ginecólogos Hospital Infantil L.V.S.
}

El trabajo de parto que no progresa con una velocidad normal se denomina disfuncional.

En la terminología clásica se emplea la palabra distocia, derivada del griego: dys, mal y tokos, parto. Algunos otros sinónimos para referirse a la misma situación incluyen parto prolongado o anormal, inercia uterina o parto variable.

La principal obligación del médico frente a un trabajo de parto disfuncional es diferenciar la disfunción del miometrio de los trastornos mecánicos.

La mayoría de las pacientes en trabajo de parto evolucionan de forma normal llegando a tener un parto vaginal espontáneo. El trabajo de parto normal se caracteriza por el progresivo borramiento y dilatación del cérvix uterino asociado con el descenso de la presentación fetal (2).

La desproporción cefalo-pélvica se define como la incapacidad de la parte que se presenta de la cabeza fetal para pasar a través de la pelvis materna.

La desproporción cefalo-pélvica es responsable de la mayoría de los trabajos de parto anormales, si la actividad uterina medida con un monitor de presión intrauterina 
es normal. Ella ocurre en aproximadamente el uno a tres por ciento de todas las primigestantes y en el $30 \%$ de aquellas que presentan alargamiento de la fase activa de la dilatación o el descenso y en el $50 \%$ de las que presentan detención en el descenso.

Si la desproporción cefalopélvica no es identificada y algún tipo de tratamiento instaurado rápidamente puede conducir a un aumento tanto en la morbilidad como en la mortalidad materna, fetal o neonatal.

El manejo adecuado de la desproporción cefalopélvica incluye su reconocimiento temprano durante el trabajo de parto, identificación de la causa y pronta instauración de una terapia. Los doctores Caldwell y Moloy en sus trabajos, considerados pioneros en la Obstetricia, lograron cristalizar el pensamiento obstétrico en cuanto a la relación existente entre la arquitectura pélvica humana y el progreso del trabajo de parto (3).

Sin embargo, el conocimiento de la relación entre la arquitectura pelviana y la posición de la cabeza fetal, unido a la evaluación de las curvas de trabajo de parto, a menudo permite una predicción o explicación útil de un trabajo de parto anormal, especialmente en la fase de descenso.

Para la evaluación de la arquitectura fetal y de sus relaciones con la cabeza fetal han sido utilizados a lo largo de la historia muchos métodos, a los cuales en uno u otro momento se ha dado mayor o menor credibilidad. En términos generales se puede decir que existen dos formas de hacer dicha evaluación: la primera de ellas es clínica y puede ser dividida a su vez en dos tipos, la pelvimetría externa y la pelvimetría interna y la segunda, se apoya en los datos obtenidos de exámenes paraclínicos tales como radiografías y más recientemente en la ecografía, tomografía axial computarizada y resonancia magnética.

\section{Sujetos y métodos}

Para el estudio se tomaron todas las pacientes que fueron admitidas en el servicio de Consulta Externa de Ginecoobstetricia del Hospital Infantil Universitario "Lorencita Villegas de Santos", que fueran nulíparas y que no cursaran con enfermedades intercurrentes con el embarazo.

A estas pacientes se les hizo la historia obstétrica utilizada en el servicio de Ginecoobstetricia. Cuando acudieron a control prenatal y cursaban con 36 a 40 semanas de embarazo se les practicó la pelvimetría clínica. Los médicos responsables de la atención del parto no conocieron los datos obtenidos de esta valoración.

Para la pelvimetría externa se utilizó el pelvímetro de Martín. Las medidas tomadas fueron: el diámetro biespinoso, que se extiende desde la faceta externa de la espina ilíaca anterosuperior de un lado al punto homólogo del lado opuesto; el bisilíaco o bicrestal, desde el punto más saliente del borde externo de la cresta ilíaca de un lado al otro; el bitrocantérico, entre los puntos más salientes de los dos trocánteres mayores de los fémures; el anteroposterior, llamado también de Baudelocque o conjugado externo, desde la depresión situada inmediatamente por debajo de la apófisis espinosa de la última vértebra lumbar a la cara anterior de la sínfisis pubiana.
Los diámetros transversos externos se obtuvieron con la mujer en decúbito supino mientras que para la obtención del diámetro anteroposterior se colocó a la mujer en decúbito lateral.

Forma parte de la pelvimetría externa la determinación del rombo de Michaelis, cuya utilidad es la apreciación de la simetría o asimetría pelviana, además de la valiosa orientación que brinda en los casos de estenosis pélvica. Los ángulos de dicho rombo son los siguientes: el superior, está marcado por la fosita que se encuentra por debajo de la apófisis espinosa de la última vértebra lumbar; el inferior, por el extremo superior del surco interglúteo; los laterales, por unas pequeñas depresiones de la piel que cubre las espinas ilíacas postero-superiores. Al unir estos cuatro puntos de referencia se forma un. rombo, del que se deben tener en cuenta tanto el diámetro vertical, que oscila entre 10 y $14 \mathrm{~cm}$, como el diámetro transverso cuya medida es más constante y es aproximadamente de $10 \mathrm{~cm}$.

Como parte de la pelvimetría externa también se practicó la medición del diámetro biisquiático y la apreciación de la forma de la arcada pubiana. Para la obtención de estos dos datos la paciente debe colocarse en decúbito supino. Para la medición de la distancia entre las tuberosidades isquiáticas se apoya el puño deprimiendo fuertemente la región vulvo-anoperineal, en contacto con las tuberosidades isquiáticas en ademán de penetrar en la pelvis, teniendo en cuenta que el puño que mide aproximadamente $8 \mathrm{~cm}$ no deberá ser detenido por los extremos del diámetro medido.

Para la apreciación de la forma de la arcada pubiana no se utiliza instrumento alguno; apoyando ambos pulgares contra las ramas descendentes del pubis y reuniéndolos a nivel del borde inferior de la sínfisis púbica (maniobra de moldeamiento de Sellheim), se reproduce la forma y amplitud del arco o ángulo del pubis y se obtiene una idea del diámetro transversal del estrecho inferior.

Mediante la pelvimetría interna se obtienen datos acerca del estrecho superior, del estrecho medio y del estrecho inferior, señalando que no gozan todos del mismo valor clínico.

Para esta exploración se coloca a la mujer en decúbito supino, con los muslos ligeramente flexionados y en abducción, evitando las posiciones extremas de flexión de los miembros inferiores que pueden determinar movimientos de nutación del sacro capaces de alterar los diámetros anteroposteriores.

En el estrecho superior interesa en primer término el promontorio. La identificación de éste implica la mensuración de la distancia que lo separa del borde inferior de la sínfisis púbica o diámetro promonto-subpúbico o conjugado diagonal que mide aproximadamente $12,5 \mathrm{~cm}$.

La pelvimetría de la excavación da a conocer las medidas del diámetro sacro-subpúbico, la forma del sacro y la de las paredes laterales. El diámetro anteroposterior de la excavación mide $12 \mathrm{~cm}$, pero más importante que éste es determinar la curvatura del sacro, que normalmente es algo cóncava. Si se encuentra que es aplanada o convexa es sugestivo de cierta estrechez del espacio disponible en sentido anteroposterior. 
Las espinas ciáticas deben palparse cuidadosamente. Es imposible medir los diámetros anteroposterior o transverso del plano medio, sin embargo, se considera que las espinas ciáticas romas, apenas palpables son de mejor pronóstico que las espinas prominentes, fácilmente palpables, que pueden representar un serio obstáculo para el pasaje de la cabeza fetal a través de la pelvis.

Por último recorriendo la pared posterior de la pelvis se puede examinar la punta del coxis, su movilidad y su articulación con la última pieza sacra.

Posteriormente se revisaron las historias y se analizó la evolución del trabajo de parto, de acuerdo al partograma de Friedman incluido en la historia obstétrica y la vía del parto. En el análisis de los datos se consideró que un trabajo de parto no era normal y que esta anormalidad podía ser debida a desproporción cefalopélvica cuando se presentó alargamiento de la fase activa de la dilatación (que en el caso de una paciente nulípara corresponde a una velocidad de dilatación menor de $1.2 \mathrm{~cm}$ por hora) o detención de la misma (cuando no progresa la dilatación por más de dos horas), o cuando se presentó alargamiento del descenso (que para una nulípara debe ocurrir a una velocidad de $1 \mathrm{~cm}$ por hora) o detención de éste. Se excluyeron las pacientes que fueron a operación cesárea por una condición distinta a la desproporción cefalopélvica de causa materna.

\section{Resultados}

Se realizaron 145 pelvimetrías en igual número de pacientes que cumplieron con los criterios de inclusión. Se excluyeron 30 pacientes a las cuales se les realizó cesárea o se instrumentó el parto por motivos diferentes a la desproporción cefalo-pélvica de causa materna, que fueron operadas con este diagnóstico pero no cumplían con los criterios para diagnosticar un trabajo de parto anormal y las pacientes que no regresaron a control prenatal y cuyo parto no fue atendido en esta institución.

Desde el punto de vista de la estadística descriptiva encontramos que el valor promedio para el diámetro biespinoso fue de $22.39 \pm 1.7$, para el bicrestíleo de 26.81 \pm 1.5 , para el bitrocantérico de $29.97 \pm 1.6$, para el de Baudelocque de $18.43 \pm 1.1$ y para el ángulo subpúbico de $86.83 \pm 6.6$ grados (Figura 1). En cuanto a la distribución de frecuencias, encontramos 92 pacientes cuyo diámetro bituberoso fue evaluado como normal y 23 en las que se encontró disminuido, 111 pacientes con el promontorio no palpable y 4 en las que fue palpable, 2 pacientes en las que se consideró que las espinas ciáticas eran prominentes y 113 en las que eran intermedias o romas, 112 pacientes en las que el sacro era posterior o medio y 3 pacientes en las que era anterior y 110 pacientes en las que las paredes laterales de la pelvis fueron normales y 5 en las que se consideró que no lo eran.

No se encontró asimetría en la valoración del rombo de Michaelis en ninguna paciente.

Todos los recién nacidos pesaron entre 2500 y $4000 \mathrm{~g}$ y todos los partos fueron a término.

La media de la medida del diámetro biespinoso de las pacientes que tuvieron un parto normal fue de $22.42 \pm 1.7$ y de las pacientes a las que se les realizó cesárea fue de $22.21 \pm 1.5$ y no se encontró diferencia significativa entre los dos grupos (Figura 2).

La media del diámetro bicrestíleo para las pacientes que tuvieron parto normal fue de $26.86 \pm 1.5$ y de las que se les realizó cesárea fue $26.55 \pm 1.4$. No se encontró diferencia significativa entre los dos grupos (Figura 3 ).

Figura 1

MEDIDAS DE LA PELVIMETRIA EXTERNA

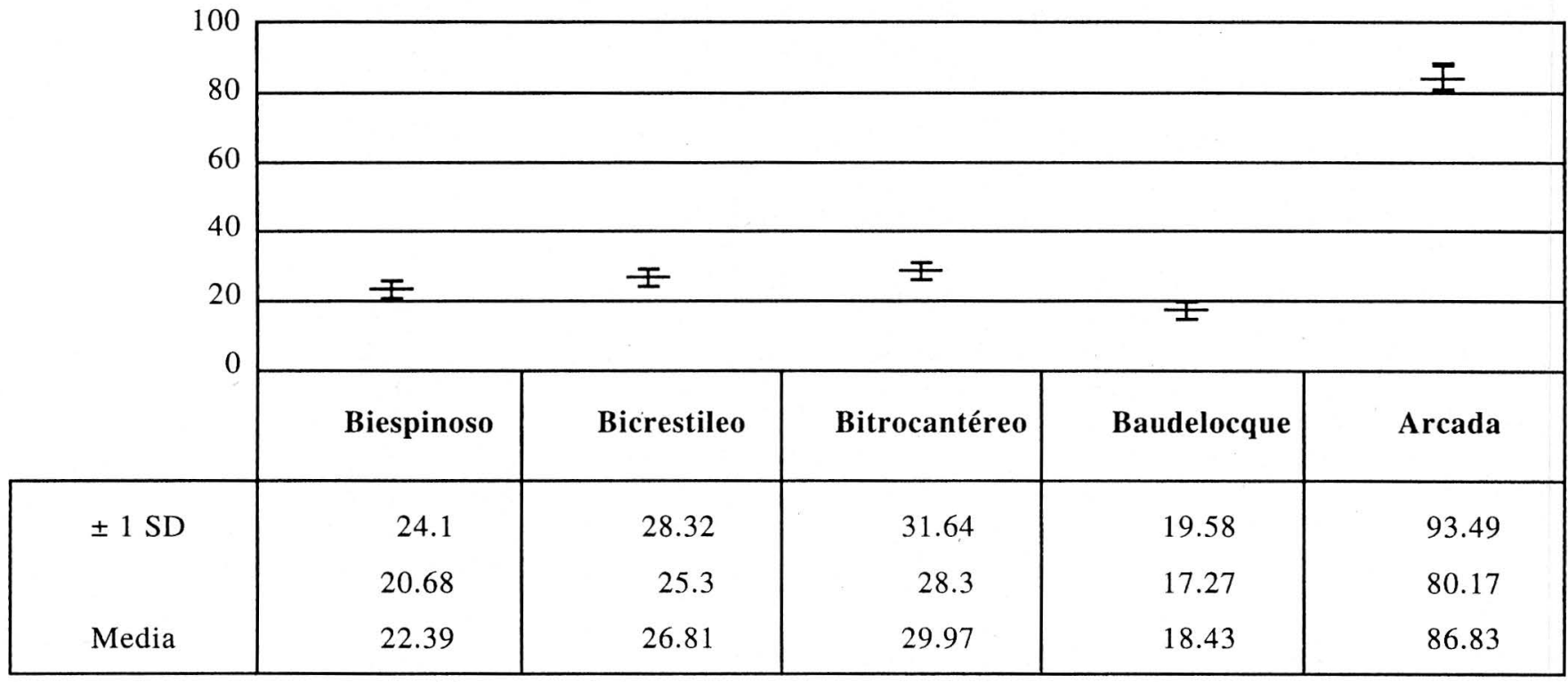

$$
I+/-1 \text { SD } \text { I MEDIA }
$$


Figura 2

\section{DIAMETRO BIESPINOSO}

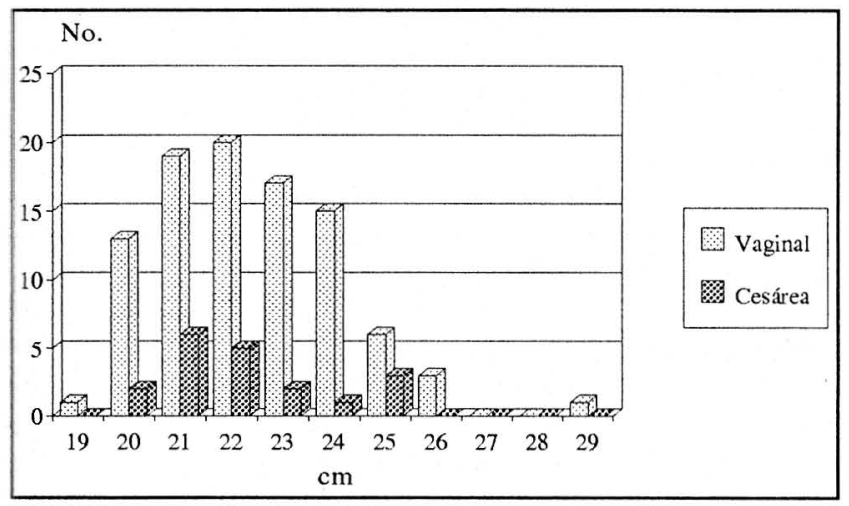

Figura 3

DIAMETRO BICRESTILEO

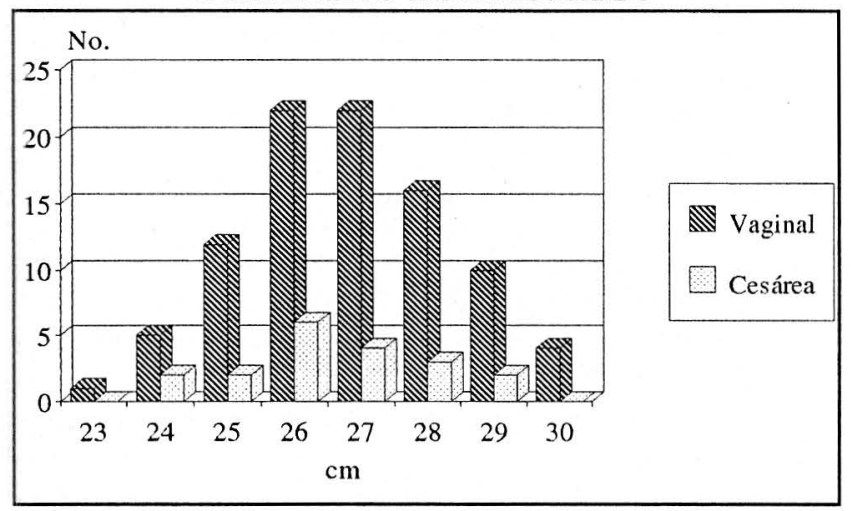

La media del diámetro bitrocantéreo fue de $29.99 \pm$ 1.7 para las pacientes con parto normal y de $29.84 \pm 1.4$ para las pacientes con cesárea. No fue significativa la diferencia (Figura 4).

La media del diámetro de Baudelocque fue de 18.46 \pm 1.1 para las pacientes con parto normal y de $18.32 \pm 1.2$ para las pacientes con cesárea. No fue significativa la diferencia (Figura 5).

La media de la medida del ángulo subpúbico fue de $86.93 \pm 6.3$ para las pacientes con parto normal y de 86.93 \pm 8.3 para las pacientes con cesárea. No fue significativa la diferencia (Figura 6).

Para realizar las comparaciones entre los dos grupos se usó el Test de $\mathrm{T}$ con dos muestras.

Se encontraron 16 pacientes con el diámetro bituberoso disminuido que tuvieron el parto normal y 7 con dicha medida disminuida a las que se les realizó cesárea; 2 pacientes con el promontorio palpable que tuvieron el parto normal y 2 pacientes con el promontorio palpable con cesárea; 2 pacientes con las espinas ciáticas prominentes que tuvieron el parto normal y ninguna paciente en el grupo de cesáreas; 2 pacientes con el sacro anterior en el grupo de parto normal y 1 paciente con el de cesárea; 2 pacientes con las paredes laterales de la pelvis evaluadas como anormales en el grupo de parto normal y 3 pacientes en el grupo de cesárea. Para analizar estos resultados se utilizaron tablas de Dos por Dos y con la
Figura 4

DIAMETRO BITROCANTEREO

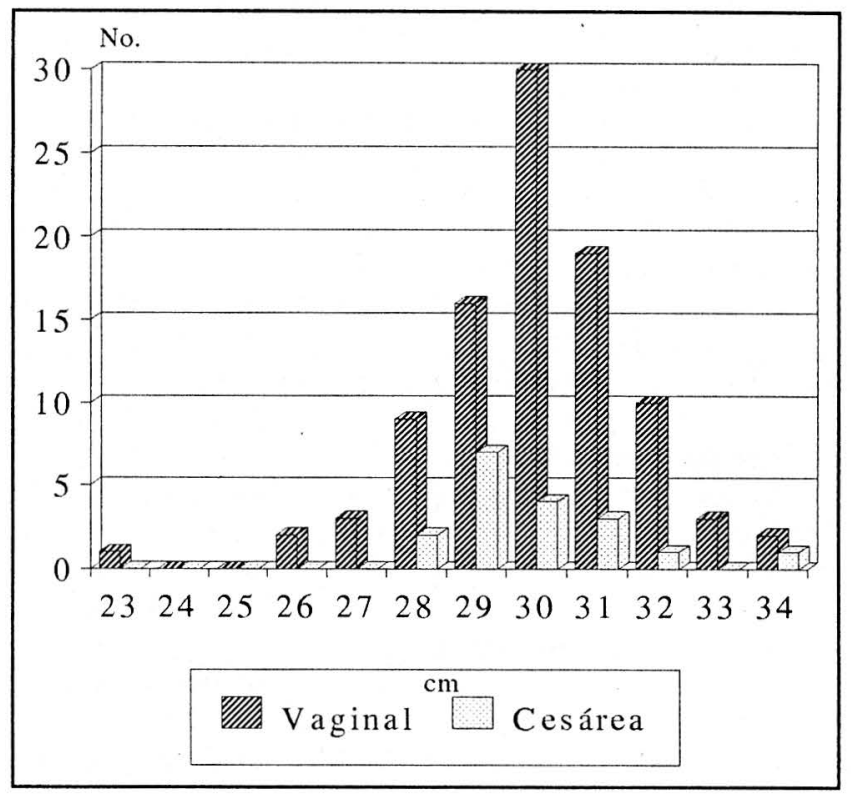

Figura 5

DIAMETRO BAUDELOCQUE

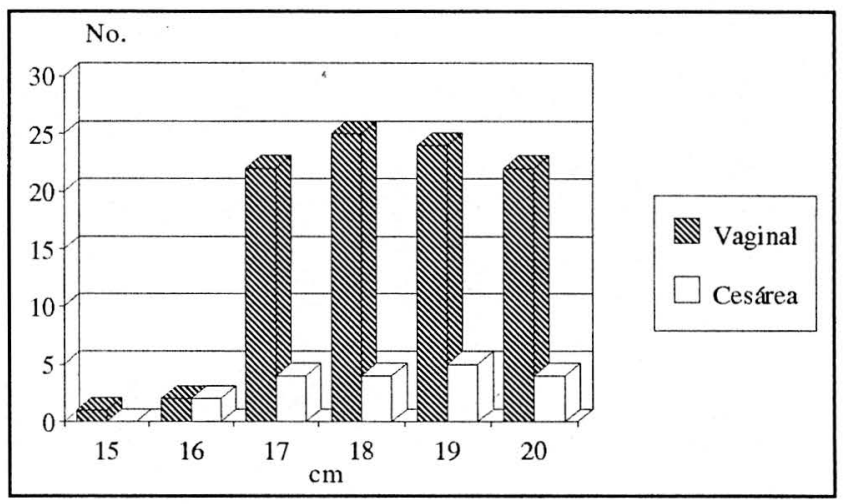

Figura 6

ARCADA SUBPUBICA

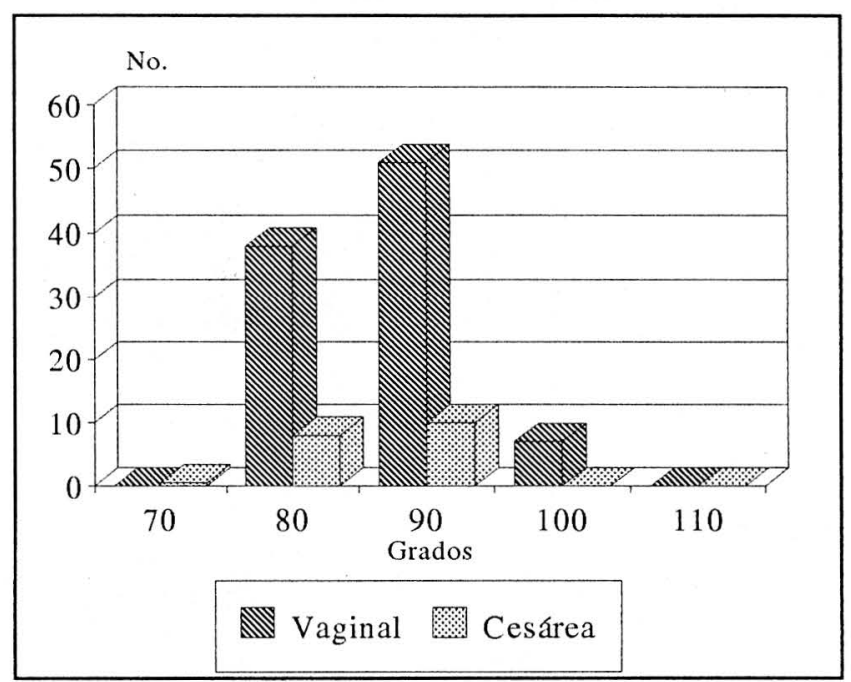


excepción de los parámetros que evaluaron el diámetro bituberoso y las paredes laterales de la pelvis, en los que se encontraron diferencias significativas $(p=0.044$ y

Tabla 1

\section{PELVIMETRIA INTERNA Y VIA DEL PARTO}

\begin{tabular}{|c|c|c|c|c|}
\hline Mediciones & Vaginal & Cesárea & Total & p \\
\hline BITUBEROSO & & & & \multirow{3}{*}{0.044} \\
\hline Normal & 80 & 12 & 92 & \\
\hline Disminuido & 16 & 7 & 23 & \\
\hline PROMONTORIO & & & & \multirow{3}{*}{0.066} \\
\hline Palpable & 2 & 2 & 4 & \\
\hline No palpable & 94 & 17 & 111 & \\
\hline ESPINAS CIATICAS & & & & \multirow{3}{*}{0.525} \\
\hline Romas o intermedias & 94 & 19 & 113 & \\
\hline Prominentes & 2 & 0 & 2 & \\
\hline $\begin{array}{l}\text { INCLINACION DEL } \\
\text { SACRO }\end{array}$ & & & & \multirow{3}{*}{0.426} \\
\hline Anterior & 2 & 1 & 3 & \\
\hline Medio o Posterior & 94 & 18 & 112 & \\
\hline $\begin{array}{l}\text { PAREDES LATERALES } \\
\text { Normales }\end{array}$ & $\mathrm{ES}$ & 16 & 110 & \multirow{2}{*}{0.007} \\
\hline Anormales & 2 & 3 & 5 & \\
\hline
\end{tabular}

0.007 respectivamente), no se encontraron diferencias significativas entre los dos grupos (Tabla 1).

Además se realizó un análisis de regresión lineal de las diferentes variables en el que se encontró que si se consideran en conjunto las medidas de la arcada subpúbica de el diámetro bituberoso, la palpación o no del promontorio y la apreciación de la forma de las paredes laterales de la pelvis se puede hacer un pronóstico acerca del tipo de parto $(\mathrm{p}=0.02)$.

\section{Discusión}

Las medidas aceptadas como normales para los diámetros biespinoso, bicrestíleo y bitrocantérico son de 24,
28 y $34 \mathrm{~cm}$ y de 19 a $20 \mathrm{~cm}$ para el anteroposterior. Estas medidas pueden tener ligeras variaciones de acuerdo con diversos factores que pueden modificar la contextura física de las mujeres en los diferentes sitios del mundo.

Es importante anotar que los valores promedio de todas estas medidas en la población estudiada se encontraron por debajo de los valores informados como normales en la literatura. Esto hace suponer que existen diferencias biométricas que se deben tener en cuenta al estudiar grupos poblacionales específicos y que estas diferencias pueden ser la consecuencia de cambios en los hábitos, en el tipo de trabajo y en la nutrición. El hecho es que desde hace unas décadas se puedan practicar cesáreas por desproporción cefalopélvica a mujeres que antes morían durante el parto o que no tenían hijos vivos, pueden haber producido un aumento en el número de mujeres con pelvis estrechas que heredaron esta condición a sus hijas.

Con los resultados obtenidos en este trabajo se concluye que no existe algún parámetro que por sí solo pueda predecir la desproporción cefalopélvica antes del inicio del trabajo de parto y que sólo si se hallan alteradas las mediciones de la arcada subpúbica, del diámetro bituberoso, de la palpación del promontorio y de las paredes laterales de la pelvis, es posible que exista una desproporción cefalopélvica de causa materna.

A pesar de que todos estos parámetros estén alterados, no se puede excluir la posibilidad de que la paciente tenga un parto vaginal normal.

El mayor conocimiento de la evolución y de la dinámica del trabajo de parto, ha permitido diagnosticar las alteraciones del parto en forma precoz y de una manera más precisa, gracias a los trabajos de Friedman y muchos otros investigadores.

Por todas las razones anteriormente expuestas, consideramos que la pelvimetría es un procedimiento clínico que brinda una gran orientación al obstetra y que lo puede alertar sobre la posibilidad de alteraciones en el trabajo de parto debidas a desproporción cefalopélvica de causa materna, pero que no puede hacer un diagnóstico absoluto de ella. Por lo tanto a todas las pacientes se les debe dar la oportunidad de tener un trabajo de parto y sólo cuando se presente alguna alteración de éste se podrá diagnosticar esta entidad con mayor certeza.

\section{BIBLIOGRAFIA}

1. Iffy L., Kaminetzky HA. Obstetricia y Perinatología. Principios Práctica. Ed. Médica Panamericana, Buenos Aires, 1985.

2. Sibai BM. Cephalopelvic disproportion, en Manual off Clinical Problems in Obstetrics and Gynecology. Little Brown and Co., Boston, 1986.
3. Eden RD. Assessment and Care of the Fetus. Appleton \& Lange, Norwalk, 1990.

4. Creasy RK., Resnik N. Maternal-fetal Medicine: Principles and Practice. W.B. Saunders Co., Philadelphia, 1989.

5. Dexeus S., Dexeus JM. Tratado de Obstetricia. Ed. Salvat, Barcelona, 1957. 